\title{
Household Wealth and Child Body Mass Index: Patterns and Mechanisms
}

\author{
COURTNEY BOEN, LISA A. KEISTER@, AND NICK GRAETZ
}

Wealth plays a unique role in shaping later-life health risk, but the relationship between wealth and child health remains largely unexplored. Using longitudinal data from the Panel Study of Income Dynamics (PSID) (1994-2013), this study uses multilevel mixed-effects models and the parametric $g$-formula approach to both assess the relationship between household wealth and child body mass index (BMI) and identify the mechanisms linking wealth to child BMI. We find that household wealth is negatively associated with childhood BMI. In addition to finding a strong, direct association, we also find that household wealth indirectly patterns child BMI and obesity risk through household spending and family stress processes. These findings provide new insights into the links between wealth, child health, and early-life population health disparities.

Keywords: wealth, child health, health disparities

Decades of research from across disciplines provides clear and convincing evidence of a socioeconomic gradient in health (Marmot 2004). Beginning at birth, the association between socioeconomic status (SES) and health is strong and positive, with babies born into socioeconomically disadvantaged households being at higher risk of preterm birth and low birthweight than babies born into higher-SES house- holds (Martinson and Reichman 2016). Physical limitations and developmental delays and disabilities are more likely for children in low-SES households and families than for children from more socioeconomically advantaged households. For example, evidence indicates that children in low-SES households are at much greater risk of being overweight or obese or experiencing hearing and vision problems,

Courtney Boen is assistant professor of sociology, research associate in the Population Studies Center and Population Aging Research Center, and senior fellow at the Leonard Davis Institute at the University of Pennsylvania, United States. Lisa A. Keister is professor in the Department of Sociology and Sanford School of Public Policy and faculty research scholar of the Duke University Population Research Institute at Duke University, United States. Nick Graetz is a PhD candidate in demography and sociology in the Population Studies Center at the University of Pennsylvania, United States.

(c) 2021 Russell Sage Foundation. Boen, Courtney, Lisa A. Keister, and Nick Graetz. 2021. “Household Wealth and Child Body Mass Index: Patterns and Mechanisms." RSF: The Russell Sage Foundation Journal of the Social Sciences 7(3): 80-100. DOI: 10.7758/RSF.2021.7.3.04. Direct correspondence to: Courtney Boen at cboen @upenn.edu, 232 McNeil Building, 3718 Locus Walk, Philadelphia, PA 19104, United States; Lisa A. Keister at Ikeister@duke.edu, 268 Soc/Psych Bldg, Durham, NC 27708, United States; and Nick Graetz at ngraetz@sas .upenn.edu, 249-2 McNeil Building, 3718 Locus Walk, Philadelphia, PA 19104, United States.

Open Access Policy: RSF: The Russell Sage Foundation Journal of the Social Sciences is an open access journal. This article is published under a Creative Commons Attribution-NonCommercial-NoDerivs 3.0 Unported License. 
asthma, and ear infections than children in higher-SES households (Larson and Halfon 2010). Importantly, studies drawing from the life course perspective demonstrate that child socioeconomic exposures not only affect health during childhood, but also shape patterns of health and disease risk across the life span (Kuh et al. 2003), well into old age (Yang et al. 2017), suggesting that the drivers of adult socioeconomic disparities in health and mortality emerge early in the life course.

Scholars increasingly point to the important role of wealth in shaping individual health and contributing to population health inequality (Boen, Keister, and Aronson 2020; Boen and Yang 2016; Pollack et al. 2007). Most research on the socioeconomic gradient in health relies on indicators of income or education as markers of SES, but evidence shows that wealth plays a unique and important role in shaping. life course patterns of individual and population health, net of other indicators of SES (Boen 2016; Boen and Yang 2016; Erixson 2017). Studies typically operationalize wealth as total net worth, or the sum of household assets minus debts. Much of what is known about the wealth-health connection comes from studies with older adult samples, but recent quasiexperimental evidence suggests that wealth may also be causally related to child health outcomes (Cesarini et al. 2016). Given increasing levels of wealth inequality in the United States in recent decades (Keister 2014) - including particularly stark racial wealth gaps (Killewald, Pfeffer, and Schachner 2017), low levels of wealth accumulation among households with children (Gibson-Davis and Percheski 2018), and high net worth poverty among households with children (Gibson-Davis, Genettian, and Keister 2020)-understanding the role of wealth in shaping patterns of child health inequality is of critical scientific and policy importance.

Still, the relationship between wealth and child health remains largely unexplored. Household wealth levels have been linked to adult health outcomes, but little is known about how wealth is associated with the health of children. This gap is particularly notable given that wealth inequality is especially high among child households (Boshara, Emmons, and Noeth 2015) and that families with children in the United States have comparatively weak social safety net protections (Gibson-Davis and Percheski 2018). The mechanisms linking household wealth to health are also unclear. Research on adults suggests that stress, health behaviors, and consumption may link wealth to health indirectly (Berger and Houle 2019; Boen and Yang 2016), but few studies formally test these mechanisms or consider how the pathways relating wealth to child health may differ from the mechanisms linking wealth to adult health outcomes. Finally, concerns about causal inference-including issues related to reverse causality and time-varying confounding-continue to plague the literature in this area, which further limits understanding of the linkages between wealth and well-being. Together, these gaps suggest that more research on the links between wealth and child health is needed.

Using nationally representative, longitudinal data from the Panel Study of Income Dynamics (PSID), this study is among the first to assess the prospective links between household wealth and child body mass index (BMI), a critical marker of child well-being. Our analyses also included other markers of child health, but we focus on BMI for substantive and empirical reasons. Substantively, childhood obesity is an early-life risk factor for a number of later-life health outcomes. The early onset of obesity threatens cardiovascular health, risk for diabetes, life expectancy, and other outcomes across the entire life course (Park et al. 2012). Obesity risks emerge early in a child's life and continue to be consequential through the developmental years and into adulthood. Obesity is thus a particularly important indicator of child health and development that has important consequences for life course health and well-being. Empirically, unlike markers of childhood disease, which are somewhat rare among children and take time to emerge, BMI is a continuous marker of health risk. BMI trajectories can be observed across the early life span, from birth though childhood, adolescence, and young adulthood. Because we use data that includes longitudinal markers of both wealth and health, we are able to observe how changes in wealth 
relate to changes in BMI across the early life course.

Using a variety of analytic techniques-including descriptive analyses, longitudinal mixed-effects models, and the parametric $\mathrm{g}$ formula mediation approach-this study is among the first to assess the links between household wealth and child health, paying particular attention to how household wealth shapes BMI and obesity risk in childhood. This article makes three specific contributions. First, we establish the link between total household net worth and BMI. Second, we assess how different components of net worth relate to child BMI, following recent research suggesting that wealth components and health may be associated in different ways (Boen, Keister, and Aronson 2020). Finally, we examine some of the mechanisms that potentially underlie the wealth-health connection in childhood, including a variety of stress-related, behavioral, and spending pathways. Together, these three aims provide a comprehensive assessment of the wealth-health relationship focused on a critical measure of child health. Contextualized in a historical period marked by growing wealth inequality, this study provides new knowledge of the socioeconomic determinants of child health and sheds new light on the potential leverage points for closing socioeconomic inequities in child well-being.

\section{BACKGROUND}

Wealth inequality in the United States is extreme and growing. Indeed, the share of wealth held by the top 1 percent of households grew from 30 percent in 1989 to 37 percent in 2019. During the same period, the share of wealth owned by the bottom 80 percent of households declined from 20 to 13 percent (author's estimates from the Survey of Consumer Finances). The growth in wealth inequality reflects several interdependent processes, including the types of assets households own. The median U.S. household depends heavily on home equity as a source of wealth, and the dramatic decline in home values during the 2007-2009 recession reduced their net worth considerably. Many of these families have still not recovered their net worth (Pfeffer, Danziger, and Schoeni 2013). By contrast, upper-wealth families are more highly invested in the stock market, and rising stock values have meant that their total wealth has tended to rise (Piketty 2013). In the years following the recession, upper-wealth households benefited from a quick stock market recovery. Incomes have also risen for top households as the median income has stagnated; this difference has added to wealth inequality by allowing top households additional resources to save and creating resource constraints for those at the median that make saving more difficult.

Even in the context of high levels of wealth inequality, racial differences in wealth ownership are extreme. Black Americans hold roughly 5 cents for every dollar of white wealth, and more than one-third of all black households have zero or negative net worth-meaning their debts exceed the total worth of their assetsrelative to 15 percent of white households (Kochnar and Cilluffo 2017). Scholars trace the historical origins of the racial wealth gap in the United States to chattel slavery and the failure of the Freedmen's Bureau and the Homestead Act to provide land to formerly enslaved people during the Reconstruction era (Darity and Mullen 2020). The forced seizure of black assets through racial violence and terrorism, the exclusion of black Americans from receiving benefits offered through the New Deal, the unequal distribution of G.I. Bill benefits in the years following World War II, and the proliferation of Jim Crow policies all contributed to the racial patterning of wealth in the United States throughout the nineteenth and twentieth centuries (Oliver and Shapiro 2013). Contemporary discrimination in the housing, lending, and labor markets further exacerbates racial wealth inequality by restricting black families' access to the same instruments of wealth accumulation as whites (Hamilton and Darity 2010).

Evidence of widening wealth inequality among households with children is growing (Gibson-Davis and Hill 2021, this issue; GibsonDavis and Percheski 2018). Prior to the Great Recession, the median net worth for child households was roughly half that among households without children (Pfeffer, Danziger, and Schoeni 2013). The tremendous wealth losses associated with the Great Recession disproportionately affected households with children (Pfeffer, Danziger, and Schoeni 
2013), with evidence showing that nonwhite, younger, and lower-SES child households experienced particularly large relative wealth losses (Boshara, Emmons, and Noeth 2015; Pfeffer, Danziger, and Schoeni 2013), which further exacerbated wealth inequality among America's children.

Although most research on the socioeconomic gradient in health comes from data on adult samples, evidence also shows that children from low-SES households experience greater risk of physical, psychological, and physiological health problems (Chen, Martin, and Matthews 2006; Larson and Halfon 2010) and more negative health shocks and events (Currie and Stabile 2003) than more socioeconomically advantaged children. Importantly, the racial stratification of socioeconomic resources in the United States shapes the racial patterning of child health and is a key mechanism linking racism to population health disparities. Relative to white children, African American children are more likely to experience socioeconomic hardship and disadvantage, which contributes to the emergence of racial health disparities in early life.

Income and education are the most widely used indicators of SES in health research on both adults and children, but it is increasingly clear that wealth plays a unique and critical role in shaping individual and population health, net of other SES indicators (Boen and Yang 2016; Pollack et al. 2007). Wealth can be a financial safety net for households during periods of economic hardship brought on by unemployment or illness; households can draw on their wealth to continue paying rents or mortgages, car payments, and other bills even when wages are temporarily suspended (Spilerman 2000). Wealth accumulation is also a status attainment process. Families accumulate wealth to make human capital investments, establish a desired standard of living, and pass along class status to descendants (Oliver and Shapiro 2013). Because of the racial patterning of wealth in the United States, evidence shows that racial wealth inequality contributes to populationpatterns of black-white health disparities (Boen 2016; Boen, Keister, and Aronson 2020; Bond Huie et al. 2003).

Research on the relationship between wealth and health has generally focused on the links between total wealth-typically operationalized as net worth, or the sum of assets minus debts - and health, but recent evidence suggests that the components of wealth relate to health in different ways (Boen, Keister, and Aronson 2020). Although the mechanisms linking various wealth components to health are largely untested, it is likely that liquid assets with more immediate cash values, such as savings and stocks, may help families pay for basic health needs such as food, housing, and transportation and afford preventative, routine, and emergency medical care. By contrast, more illiquid assets, such as homes and equity, may promote health by conferring psychosocial benefits to owners, including high levels of control and stability (Boen, Keister, and Aronson 2020). Home equity and value may also be proxies for other unobserved factors that shape child health, such as school and neighborhood quality. Additionally, whereas assets protect health, high levels of debt-particularly unsecured debt-increase feelings of hopelessness and frustration and overall stress burden in ways that negatively affect health (Berger and Houle 2019). For these reasons, research should not only consider the associations between total wealth and health, but also assess how the associations between wealth components and health vary.

Research on the wealth-health connection largely relies on older adult samples, but a recent study from Sweden provides evidence of a potential causal link between wealth and child health. Using administrative data on Swedish lottery players, David Cesarini and colleagues (2016) find that increases in household wealth reduce childhood obesity risk and increase child health utilization in ways that may be protective of health. The effect sizes in the study were modest, but the authors note that the impacts of wealth on child health may be greater in other national contexts where social safety net protections for families are weaker than Sweden, such as in the United States.

Mechanisms Linking Wealth and Child Health Because the factors patterning household wealth are structural-located in historical and present day policies and practices that un- 
equally distribute wealth-building opportunities and financial risks in the populationexamining how wealth shapes child health through household- and family-level mechanisms can provide new insights into the links between macro-level structural economic forces, meso-level household and family environments, and micro-level individual healthrelated processes. To examine the connections between wealth and child health, this study draws on the bioecological model of child development, which highlights the essential role of social contexts in shaping child health and development and suggests that more proximate contexts have the greatest impacts on child well-being (Bronfenbrenner and Ceci 1994). For children, the family context-including the family's socioeconomic resources, risk, and opportunities-is a critical meso-level environment that greatly shapes daily life in ways that affect their health. The socioeconomic resources available to families and households influence child well-being by affecting many of the more proximate determinants of child health and obesity risk, including household stress levels, household food budgets and spending, the availability and accessibility of opportunities and spaces for physical activity, and sleep quantity and quality (McCurdy, Gorman, and Metallinos-Katsaras 2010; Schmeer 2012). Still, given that wealth inequality in child households is so high (Gibson-Davis and Hill 2021, this issue; Gibson-Davis and Percheski 2018), it follows that children living in low- and high-wealth environments may face strikingly different household contexts that shape their health risk.

Merging insights from the bioecological models of child development, the conceptual model linking wealth to child outcomes laid out by Christina Gibson-Davis and Heather Hill in the introduction to this issue (2021), and recent work on the links between debt and child well-being by Lawrence Berger and Jason Houle (2019), we hypothesize that two primary mechanisms link household wealth and child health. First, guided by the stress process (Pearlin 1989), household wealth may affect child health through stress-related processes. In particular, we expect that low levels of household wealth produce economic pressures, anxieties, and conflict among parents; these stresses, in turn, affect child health outcomes both directly through physiological processes and indirectly through behavioral mechanisms. We hypothesize that parental and household stress can affect child health risk directly through a variety of biophysiological processes. In response to stress, the body upregulates activity in the sympathetic nervous system and hypothalamicpituitary-adrenal axis, which together govern a host of physiological processes. This upregulation of bodily stress response in response to acute stressors is sometimes referred to as "fight or flight" and serves an important role in protecting individuals from immediate threats or infections. However, chronic activation of these systems in response to repeated social stress exposure gives rise to a host of physiological pathologies, including the promotion and redistribution of bodily fat stores. Together, these physiological changes result in increased weight gain and visceral adiposity (Scott, Melhorn, and Sakai 2012). A number of studies link chronic stress exposure-including the stress associated with material deprivation and hardship - to increased risk of obesity and metabolic risk in both children and adults (Boen 2020; Garasky et al. 2009; Tate et al. 2015). In fact, chronic stress exposure in childhood may affect more than obesity risk in childhood: the damaging effects of stress on health during this critical stage of brain and physiological development may also affect long-term health (Yang et al. 2017). Indeed, exposure to chronic stress in childhood-including exposure to the many stressors or strains associated with low SES - can result in permanent or lasting changes to neurobiology and physiology that result in metabolic abnormalities across the life span and through late life (Tamayo, Herder, and Rathmann 2010).

Levels of household stress, which are shaped by household socioeconomic conditions, can also affect child obesity risk indirectly through behavioral mechanisms. Because of structural constraints, parents and caregivers in low-wealth households need to devote significant time, energy, and resources to managing the many strains, conflicts, and stressors associated with having low or inadequate financial resources. As a result, they 
may have fewer resources to invest in establishing health-promoting environments and behaviors in children (McCurdy, Gorman, and Metallinos-Katsaras 2010; Schmeer 2012). For these reasons, we expect that children in lowwealth households, which are characterized by greater financial strain and higher levels of familial and caregiver stress, will exhibit worse health behaviors than children in high-wealth families. Together, these direct biophysiological and indirect behavioral pathways may link the stress of low levels of wealth accumulation or high debt to child health in the short-term and increased health risk across the adult life course.

Second, household wealth may affect child health through consumption by shaping the quantity and quality of goods available to children (Berger and Houle 2019). Households with less wealth have less capital than higher-wealth families to invest in education, childcare and afterschool programs, housing, and other expenditures that affect child health and wellbeing. Because they lack the financial security and material resources afforded by wealth, lower wealth families may struggle to meet basic health needs of children; they may face difficulties securing housing, purchasing food, and paying for adequate levels of childcare. By contrast, households with more wealth may be able to spend more on household and childrelated costs in ways that promote child health and well-being. These linkages may be particularly relevant to the wealth-BMI connection because of the high cost of critical health resources needed to support healthy lifestyles and environments (Powell and Bao 2009). In these ways, the risks and constraints faced by low-wealth families-including high levels of stress and financial challenges-may undergird the links between household wealth and child BMI.

\section{The Life Course Perspective}

In addition to elucidating the drivers of child well-being, research on the links between wealth and child health expands knowledge about the early-life origins of adult health and social well-being. Evidence indicates that earlylife socioeconomic exposures shape life course trajectories of health both directly and indi- rectly through a variety of social and biological mechanisms (Kuh et al. 2003). Childhood is a sensitive period of development when social exposures-including socioeconomic exposures-can induce structural and functional biological and physiological changes that affect later-life health and disease risk (Kuh et al. 2003; Yang et al. 2017). This is particularly true in the case of childhood obesity. Childhood weight status reflects social exposures occurring in utero, during infancy, and throughout childhood (Isong et al. 2018), and childhood BMI is a widely documented risk factor for adult obesity (Ward et al. 2017). Childhood obesity has been positively associated with premature morbidity and mortality in adulthood, including increased risk of blood pressure, insulin resistance, low-density lipoprotein and total cholesterol, physiological inflammation, and coronary heart disease (Park et al. 2012; Reilly et al. 2003). In addition to their direct biophysiological impacts, childhood socioeconomic exposures can shape life course trajectories of health and well-being indirectly by affecting cognitive development, educational achievement and attainment, earnings, and other forms of human capital (Currie and Stabile 2003). Children in low-SES homes miss more days of school and experience more hospitalizations (Case, Lubotsky, and Paxson 2002) in ways that pattern their health and socioeconomic status across the life span. Evidence further suggests that upward social mobility in adulthood may not erase the health disadvantages associated with early-life socioeconomic hardship, particularly for individuals of color, who may experience especially challenging pathways toward mobility (Gaydosh et al. 2018) characterized by high levels of exposure to discrimination and stigmatization (Assari 2018). Assessing the links between household wealth and health during childhood can thus shed new light on the possible early-life social and biological mechanisms underlying adult patterns of health and well-being. Together, theory and research from the life course perspective reveal that consideration of the early-life origins of adult health risk is essential to preventing disease and population disparities before they emerge and, in many cases, diverge with age. 


\section{Research Questions}

We use nationally representative, longitudinal household- and child-level data from the PSID to study the association between household wealth and child BMI. We use a combination of multilevel mixed-effects models and the parametric g-formula to assess the links between wealth and child health and to examine a host of stress- and consumption-related mechanisms underlying the relationship between wealth and child BMI and obesity risk. Together, these methods allow us to estimate the direct and indirect pathways linking wealth and child BMI and obesity risk in a longitudinal, counterfactual causal inference framework. We ask three questions:

Does household wealth relate to BMI and obesity risk during childhood?

Do various components of wealth-including savings, stocks, home equity, and debtdifferentially relate to childhood BMI and obesity risk?

What are the mechanisms linking household wealth to childhood BMI?

\section{DATA AND ANALYTIC METHODS}

As noted, our data come from the Panel Study of Income Dynamics, the longest running nationally representative, longitudinal survey of individuals and families in the United States. The PSID has interviewed respondents and their family members continuously since 1968 , including biennial interviews since 1997 . We use the main PSID file, the Child Development Supplement (CDS), and the Transition to Adulthood Supplement (TAS). The CDS is a longitudinal study of children who were twelve years old or younger in 1997 and followed over three waves (CDS-1 in 1997, CDS-2 in 2002-2003, and CDS-3 in 2007-2008). The TAS began in 2005 and followed children from the original CDS cohort into young adulthood. Data on our outcomes come predominately from the CDS, and we use data from the TAS $(2009,2011,2013)$ to follow the CDS respondents who entered the CDS as infants and young children and who were followed in the TAS until they are eighteen. We link data on the CDS and TAS children to family and household characteristics available in the main file of the PSID, as well as household wealth data from the PSID wealth supplement. The data are from six survey waves (1994, 1999, 2005, 2007, 2009, and 2011) of the main PSID file that include information on family and household characteristics. These data predate the CDS data to enable lagged wealth measures.

The 2007-2009 recession, an exogenous shock to household wealth, improves our ability to make inferences about the relationship between wealth and health. Concerns about endogeneity are a major challenge to scholarship on the links between wealth and health. To improve our ability to make inferences about the relationship between wealth and health, we leverage the Great Recession's exogenous shock to household wealth. Our period of observation includes the years leading up to (1997-2005), during (2007-2009), and after (2011-2013) the recession. By CDS III (2007-2008), many households-particularly black and Hispanic households (Pfeffer, Danziger, and Schoeni 2013) and households headed by younger adults (Boshara, Emmons, and Noeth 2015)—experienced significant wealth losses as a result of the recession. During the Great Recession, households lost wealth through market mechanisms such as the "bursting" housing bubble-rather than as a consequence of changes to individual health. Documented links between wealth and health observed during this period are therefore less likely to be subject to concerns about reverse causality and endogeneity. We take empirical advantage of this shock to household wealth and the ensuing economic downturn to better isolate the relationship between wealth and child health.

\section{Outcomes: Childhood BMI}

Our outcome variable is body mass index (BMI). Research across disciplines links childhood BMI and health risk in both childhood and adulthood. Whereas measures of disability, disease, or mortality are widely used in studies of adults, BMI reflects continuous changes in well-being that can be measured in childhood and across the life span, making it particularly well suited for studying age trajectories of 
health (Deaton and Paxson 1998; Shaw and Krause 2002). In preliminary analyses, we also studied several other child health outcomes, including a count index of chronic conditions, a continuous depressive symptoms score, and markers of child self-rated health and caregiverrated health. However, few children had any chronic conditions, elevated depressive scores, or poor rated health. Without enough variation on the dependent variables, we could not assess whether or how wealth is associated with these outcomes. By contrast, BMI trajectories can be observed across childhood, and social gradients in BMI emerge early in the life span, serving an important role in shaping long-term health across a number of cardiometabolic outcomes. High BMI puts children at risk of future health problems. In this study, we operationalize childhood BMI two ways. First, we include a continuous measure of BMI $\left(\mathrm{kg} / \mathrm{m}^{2}\right)$. Second, we include a measure of age-specific BMI $\mathrm{z}$ score, which indicates where respondents fall on the BMI distribution at every age during. childhood. The continuous measure allows us to assess how wealth shapes trajectories of BMI; age-specific z-scores allow us to better understand how wealth relates to childhood overweight and obesity risk. To measure BMI, PSID interviewers measured child height and weight during the CDS-TAS interviews. Both BMI measures are time-varying indicators calculated using the measured height and weight at each wave.

\section{Key Exposures and Mechanisms}

The key exposure of interest is total household wealth, a continuous measure that reflects household net worth (a sum of household assets minus debts). We adjust the wealth measure for inflation (measured in 2017 dollars) and household size. Household wealth is included as a time-varying, normalized, lagged measure so that child health at time $t$ is modeled as a function of wealth at time $t-1$. In addition to assessing the links between total household wealth and child health, we also examine how various components of wealth relate to child BMI and obesity risk. To do so, we create measures of home equity, stocks and bonds, checking and savings, value of other as- sets, and total debt, all included as continuous, inflation-adjusted, lagged measures. We also include a measure of home value, which we include as a percentile of the PSID sample distribution, given the extreme skew of home values in the sample. Finally, we include a dummy indicator for whether the family owns a home. Supplemental analyses with alternative operations of all of the wealth variables (inverse hyperbolic sine transformed, squared measures, spline regression with varying numbers of knots to test for nonlinearities, and so on) yielded substantively similar results to those reported here.

An alternative specification of these models might include separate indicators for liquid (for example, cash accounts such as checking accounts, other financial assets) and illiquid assets (the family home, business assets, other tangible assets). We explored breaking wealth into liquid and illiquid assets, but, consistent with previous work (Boen, Keister, and Aronson 2020) our analyses showed that using home equity, stock and bond value, the value of checking and savings accounts, the value of other assets, and total other debts is a better representation of the wealth ownership of the households we study. This is consistent with research showing that the primary residence (an illiquid asset) is the most significant asset a median household owns and that stocks (a liquid asset) are becoming more commonly held among median households, particularly in retirement accounts. Other illiquid assets (such as businesses) are not commonly held by the median household and make little difference in our models.

To model the direct stress-related mechanisms, we include two measures of familial stress. First, parenting strain is measured using the Aggravation in Parenting Scale of the CDS. The CDS asked parents or primary caregivers of CDS respondents nine questions about their level of parenting stress (for example, feeling trapped by responsibility, feeling tired from raising a family). The final scale is an average score of their responses to the nine items. Second is family economic strain. The CDS asked parents or primary caregivers fifteen yes or no questions reflecting different dimensions of 
economic strain (for example, postponed major purchase because of economic problems, fallen behind in paying bills). Our final scale is the sum of binary responses to the questions. The inclusion of the economic strain measure allows us to examine how low wealth may create financial stress in parents in ways that shape child health. Further, inclusion of the parenting strain allows us to capture how the constraints and challenges of low wealth may have spillover consequences for other domains of family stress. As described, because of structural constraints, parents in low-wealth households may need to devote more time and energy to dealing with the challenges of financial insecurity-including securing housing and figuring out how to pay bills-in ways that restrict the time or resources they are able to devote to other domains. In this way, low wealth may be a primary stressor in the lives of lowwealth parents, but may also give to a host of secondary stressors, including parenting strain (Pearlin et al. 1990), in ways that pattern child health.

To assess whether wealth shapes child health through child behavioral mechanisms, we include indicators of child physical activity (total minutes of physical activity per week), fruit and vegetable consumption (sum of number of days in the last week respondent ate fruits and respondent ate vegetables), and sleep (average number of hours per night). All of these behaviors are highly associated with child BMI and obesity risk and thought to be key mechanisms linking familial financial stressors and constraints to child health.

Finally, we include four spending-related mechanisms, including indicators of educational spending, which measures all schoolrelated educational expenditures; childcare spending; health spending, which includes expenditures for hospitals and nursing homes, doctors, prescription drugs, and insurance; and housing spending, which includes mortgage and loan payments, rent, property tax, insurance, utilities, cable television, telephone, internet charges, home repairs, and home furnishings. Families can use accumulated wealth to make human capital investments in children, establish a desired standard of living, and pass along class status to descendants (Oliver and Shapiro 2013). Households with more wealth may be able to invest more in their children's education, their childcare, their health care, and their housing in ways that shape children's long-term trajectories of health. Each of these measures indicated annual household spending on the respective categories. Across a variety of model specifications, only education spending was significantly associated with child BMI and obesity risk. For this reason, in our final models, we include only education spending as a consumption-related mechanism. Supplementary analyses operationalizing the spending variables as proportions of total annual income or wealth produced substantively similar results.

\section{Other Measures}

To assess racial disparities in household wealth and child health, we include a measure of parent-primary caregiver race $(1=$ nonHispanic white; $2=$ non-Hispanic black; $3=$ other). Among children in the CDS, levels of match between parent-caregiver and child race are high; fewer than 4 percent of cases were discordant. In the majority of discordant cases, parent-caregiver race was white or black, and child race was marked as other. Supplementary analyses using child race yielded substantively similar results. Because of tremendous racial disparities in household wealthcorresponding to parent-caregiver race, rather than child race-we use parent-caregiver race in our final analytic models. To test for differential associations between wealth and child BMI by race, we ran supplementary models that included an interaction for race ${ }^{*}$ wealth, but the interaction term was not statistically significant and thus was excluded from final models. Our models include a number of other covariates reflecting child sociodemographic characteristics, including age (continuous), gender $(1=$ male $)$, and birth order. We also include a number of household characteristics, including parental-caregiver marital status (1 $=$ married $)$, maternal education $(1=$ high school or less, 2 = some college, 3 = bachelor's degree or higher), annual household income, and child health insurance status $(1=$ insured $)$. Supplementary analyses also adjusted for parental age, which was not associated with the 
outcomes and excluded from final model estimates.

\section{Analytic Sample}

The analytic sample includes respondents from the original CDS-1 followed through age eighteen. We include respondents with at least one observation. The mixed-effects models assessing the associations between total wealth, the wealth components, and the outcomes use the full sample of CDS and TAS respondents ( $\mathrm{n}=$ 2,271, 5,411 person-years). To handle missing data, we used multiple imputation by chained equations procedures with thirty imputations and thirty burn-in iterations (van Buuren and Groothuis-Oudshoorn 2011).

\section{Analytic Strategy}

We first present descriptive analyses for the full sample. Although we do not explicitly model racial disparities in BMI, we also show blackwhite differences in household wealth to highlight the particularly stark levels of black-white wealth inequality among child households in the PSID.

Next, we use longitudinal mixed-effects models to estimate the associations between total household wealth, the wealth components, and childhood BMI and obesity risk as children age. In these models, observations at level 1 are nested within individuals at level 2 (Raudenbush and Bryk 2002). The models include random intercepts and clustered standard errors at household level. We model BMI and BMI z-scores as linear outcomes. These models proceed in a stepwise fashion. Model 1 includes all child sociodemographic characteristics, family structure, and socioeconomic factors but does not include any of the wealth measures. Models 2 through 5 build on model 1 by including the wealth measures: model 2 includes total wealth; model 3 includes continuous measures of home equity, debt, stocks, checking and savings, and other assets; model 4 includes an indicator for homeownership; and model 5 includes home value. Together, results from these models show how the measures of household wealth relate to child health across childhood. Supplemental analyses including wealth*age interactions revealed that the associations between wealth and BMI were consistent with age, so the interactions were excluded from the final models.

Finally, we use the parametric g-formula (Keil et al. 2014; Naimi, Cole, and Kennedy 2017) to estimate how wealth relates to child health over time both directly and indirectly through our hypothesized mechanisms. This recently developed methodology in epidemiological causal inference allows us to parameterize the contributions of wealth and the stress, behavioral, and spending mechanisms to child BMI over time under a less restrictive set of confounding assumptions than conventional regression adjustment. In estimating the proportion of the total effect explained by a mediating variable, conventional regression conditions on measured confounders of the exposureoutcome relationship and assumes that no mediator-outcome confounders are affected by the exposure of interest (such as family wealth). However, if a variable both confounds the mediator-outcome relationship and acts as a mediator between the exposure and the outcome, it is not possible in a regression setting to control for the confounding pathways and not simultaneously overcontrol the mediating. pathways. This issue can become compounded over repeated observations of the exposure and mediators, often leading to biased estimates of the mediating effect of a specific variable (Naimi, Cole, and Kennedy 2017). For example, in our study, parental strain may influence wealth, child BMI, and other mediators of wealth-health (for example, economic strain); at the same time, wealth may influence child BMI through patterning parental strain over time. In this study, we use the g-formula approach to estimate the pathways through which household wealth shapes child BMI directly as well as how its impacts might accumulate indirectly and manifest through other time-varying characteristics across early and middle childhood and adolescence. Figure 1 provides a generalized illustration of this approach. We estimate models at three developmental periods: early childhood (ages three through seven), middle childhood (ages eight through twelve), and adolescence (ages thirteen through seventeen). In each set of models, we include timevarying characteristics that serve as both confounders and mediators in the relationship 


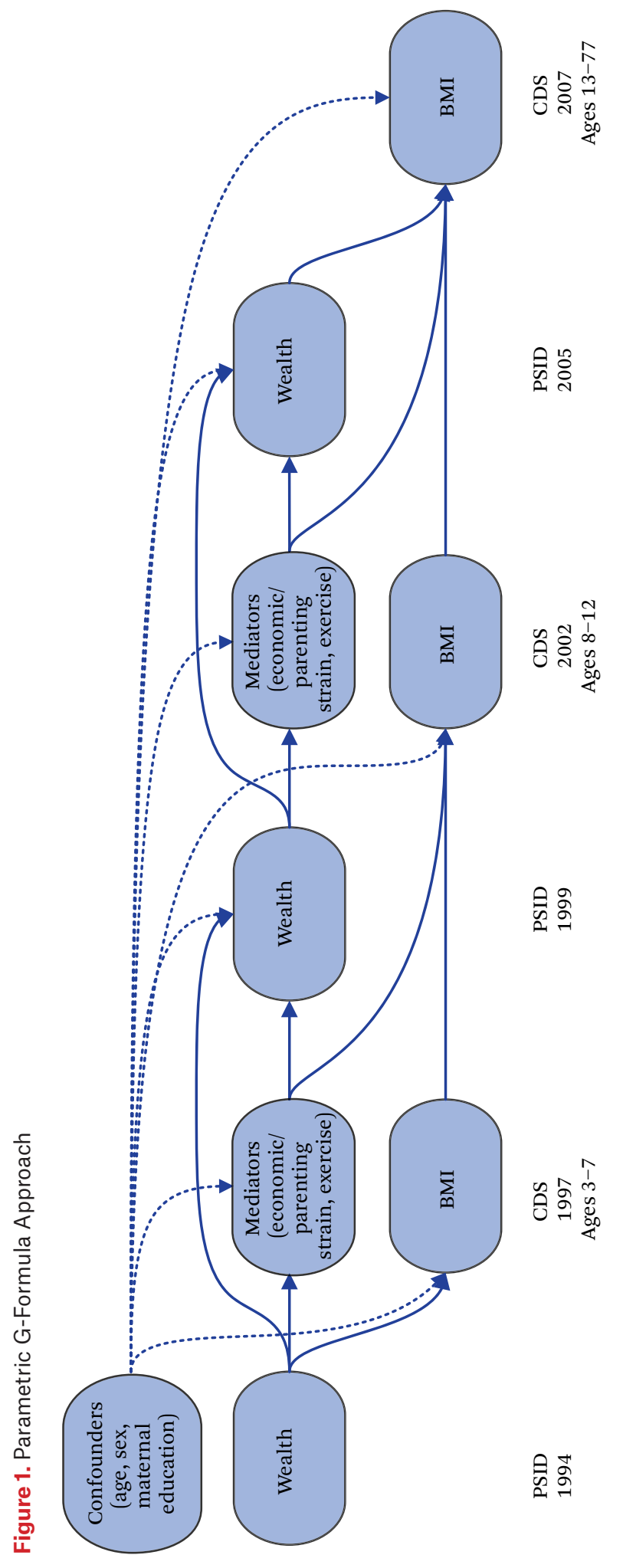

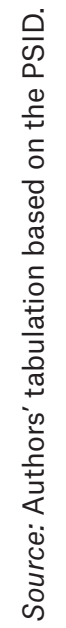


Table 1. Descriptive Statistics

\begin{tabular}{|c|c|c|}
\hline & Mean/Proportion & $\begin{array}{l}\text { Standard } \\
\text { Deviation }\end{array}$ \\
\hline Child body mass index (BMI) $\left(\mathrm{kg} / \mathrm{m}^{2}\right)$ & 20.85 & (6.05) \\
\hline Child BMI age-specific z-score & 0.09 & (0.93) \\
\hline Household wealth (US\$; median) & $29,922.56$ & $(138,057.51)$ \\
\hline House value (US\$; median) & $102,932.4$ & $(1,042,752.69)$ \\
\hline Home equity (US\$; median) & $13,601.17$ & $(116,149.21)$ \\
\hline Total debt (US\$; median) & 680.06 & $(24,850.84)$ \\
\hline Total stocks (among households who own stocks; US\$; median) & $16,378.95$ & $(214,002.23)$ \\
\hline Checking and savings value (US\$; median) & $13,146.43$ & $(47,598.8)$ \\
\hline Other assets (US\$; median) & $3,538.44$ & $(214,668.29)$ \\
\hline Owns home (1 = yes) & 0.69 & () \\
\hline Owns any stocks ( $1=$ yes $)$ & 0.28 & 0 \\
\hline Parenting strain (range: 17) & 2.26 & $(0.77)$ \\
\hline Economic strain (range: 115) & 0.96 & $(1.66)$ \\
\hline Education spending (dollars) & $1,894.38$ & $(5,376.24)$ \\
\hline Physical activity (minutes per week) & 138.68 & $(88.1)$ \\
\hline $\begin{array}{l}\text { Fruit and vegetable consumption (number of } \\
\text { times ate fruit or vegetables in last week) }\end{array}$ & 8.98 & $(3.46)$ \\
\hline Sleep (hours per night) & 8.52 & $(1.71)$ \\
\hline Child age (years) & 11.51 & $(4.13)$ \\
\hline Child gender ( 1 = male) & 0.52 & () \\
\hline Child birth order (mean) & 1.58 & $(0.83)$ \\
\hline Child race (1 = black) & 0.18 & () \\
\hline Family structure (proportion married parents) & 0.75 & () \\
\hline Maternal education $(1=\mathrm{BA}+)$ & 0.25 & () \\
\hline Child insured $(1=$ yes $)$ & 0.89 & () \\
\hline Family income (US\$; median) & $36,032.39$ & $(41,946.75)$ \\
\hline Person-years & 5,411 & \\
\hline Unique children & 2,271 & \\
\hline
\end{tabular}

Source: Authors' tabulation.

Note: Household income and all wealth measures are inflation adjusted (2017 dollars); $\mathrm{n}=2,271 ; 5,411$ person-years.

${ }^{*} p<.05 ;{ }^{* *} p<.01 ;{ }^{* * *} p<.001$

between wealth and child health. In early childhood, we model BMI and the proposed mechanisms as a function of lagged family characteristics, including wealth. In middle childhood, we model BMI and the mediating mechanisms as a function of lagged early-childhood characteristics and exposures (including lagged BMI, familial stressors, health behaviors, and household spending). In adolescence, we model the outcomes and mechanisms as a function of lagged childhood characteristics and exposures. In this way, we use the g-formula to parameterize the entire "cascade" of exposures across the child life span that link household wealth to adolescent BMI.

\section{RESULTS}

Table 1 presents descriptive statistics for the full sample. Among households with children, the median wealth is $\$ 29,923$. Still, racial wealth inequality among child households is high. Figure 2 depicts racial differences in household wealth between black and white children by showing the striking overdispersion of black households with near-zero net worth relative to white households. 
Figure 2. Black-White Disparities in Household Wealth Among Child Households (PSID, 1997-2013)

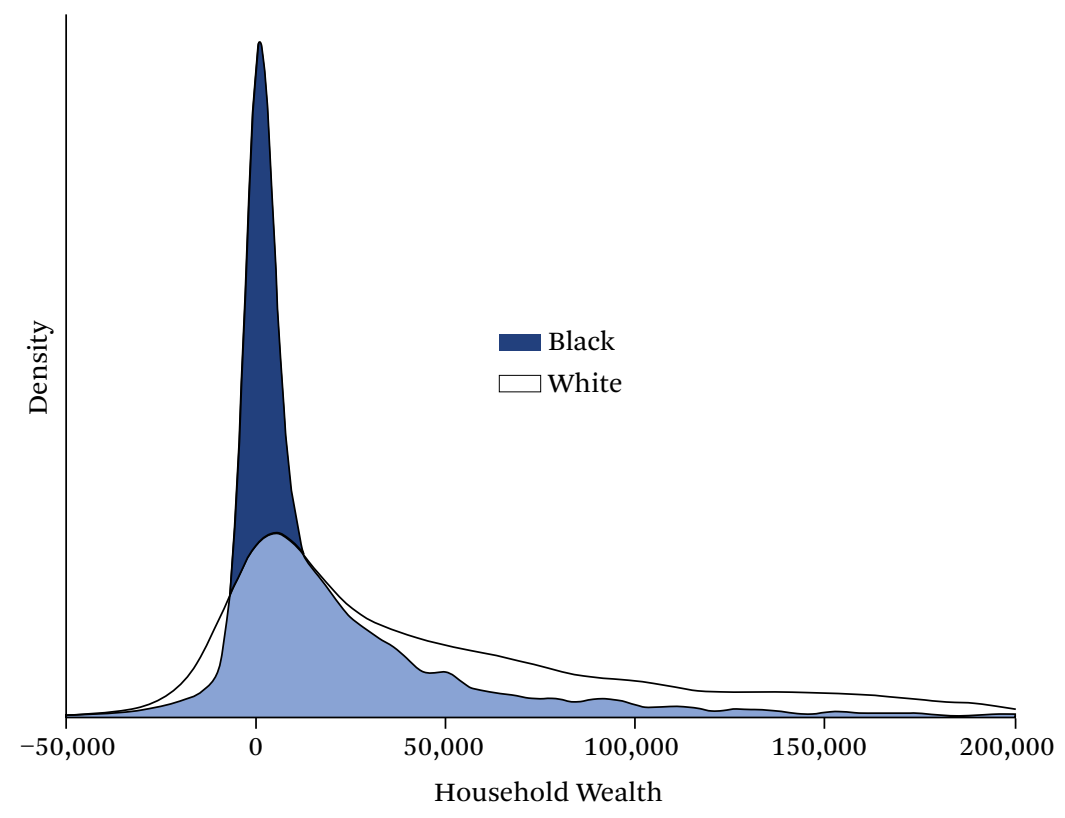

Source: Authors' tabulation based on the PSID.

Note: Household wealth is operationalized as total net worth (assets minus debts), inflation adjusted to 2017 dollars, adjusted for family size, and truncated between $\$ 150,000$ and $\$ 500,000$.

Results from longitudinal mixed-effects continuous BMI models are in table 2. Results from models using the age-specific BMI z-score outcome were substantively similar to those using the continuous BMI measure (see table A1). Model 1 is a basic model that includes all covariates except the wealth measures. Aside from child race and age, the other measures of household sociodemographic context included in model 1-including family structure, maternal education, household income, and insurance status-are not associated with child BMI. Model 2 builds on model 1 by including total household wealth and reveals that household wealth is negatively associated with child BMI $(-0.35, p<.05)$, net of other indicators of household SES including household income and maternal education. We document a similar association in model 2 of table A1, where total wealth is negatively associated with age-specific BMI z-score $(-0.07, p<.01)$. Together, results from model 2 show that household wealth is associated with child BMI and obesity risk, net of other important dimensions of household socioeconomic status. In model 3, we disaggregate the total wealth measure into continuous measures of various wealth components, including home equity, stocks, checking and savings, other assets, and wealth. Results in table 2 and table A1 indicate that home equity is associated with child BMI $(-0.27, p<.05)$ and agespecific BMI $z$-score $(-0.05, p<.01)$. We find no significant associations between debt, stocks, checking and savings, or other assets and the outcomes. In model 4 , we build on model 1 by adjusting for homeownership. We find no association between the dichotomous measure of homeownership and child BMI or obesity risk. Finally, in model 5, we build on model 1 by adjusting for home value and find no association between home value and child BMI or obesity risk.

Results of the parametric g-formula mediation analyses are presented in table 3 . In these models, we decompose the disparity in average adolescent BMI between respondents at -1 standard deviation (SD) below mean household wealth and +1 SD above the mean. We estimate 


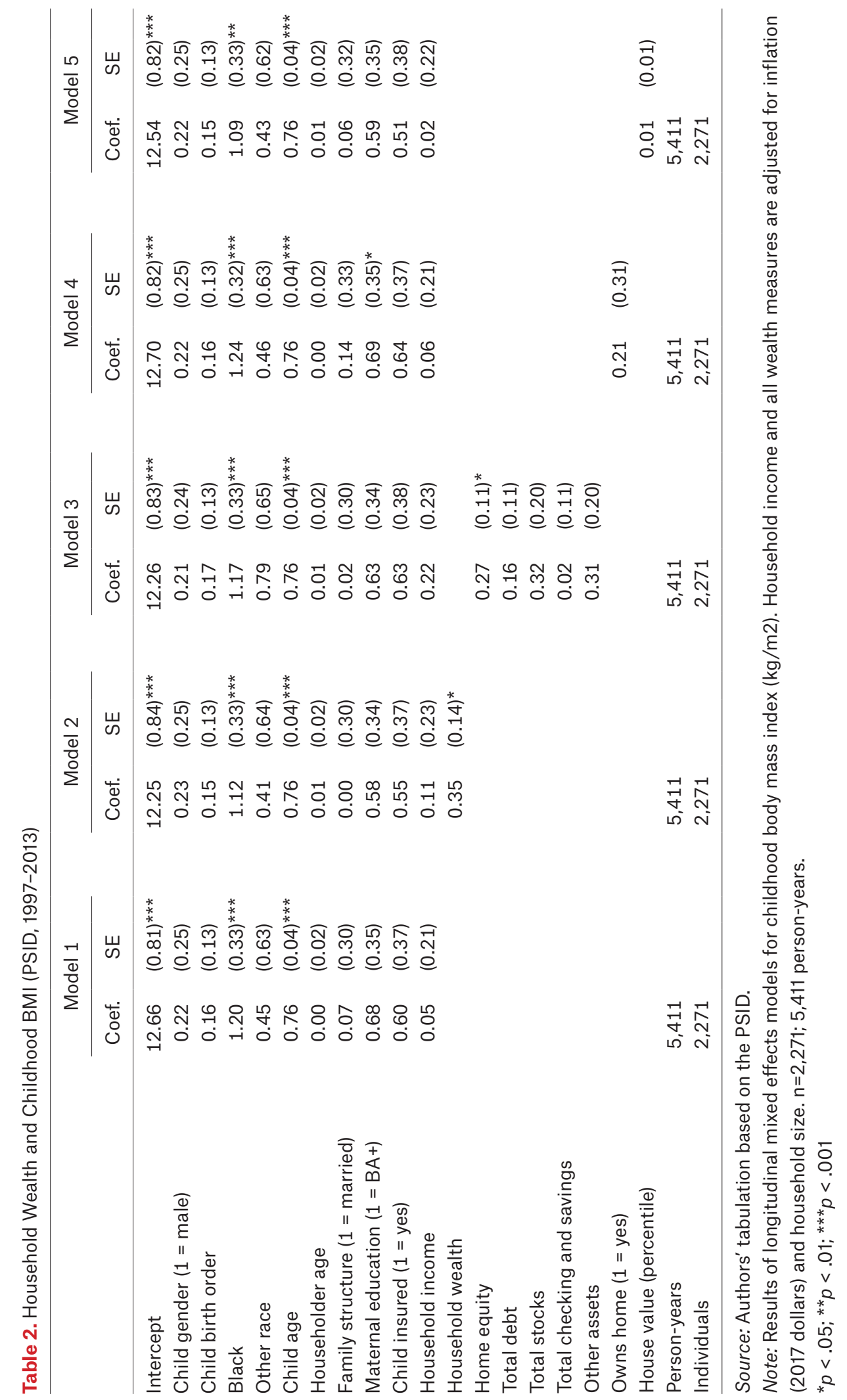


Table 3. Mediation Results: Parametric G-Formula

\begin{tabular}{|c|c|c|c|c|c|c|c|c|}
\hline & \multicolumn{4}{|c|}{ Model 1} & \multicolumn{4}{|c|}{ Model 2} \\
\hline & Coef. & SE & & Explained & Coef. & SE & & Explained \\
\hline Total effect & 1.39 & $(0.61)$ & $* *$ & $100 \%$ & 0.25 & $(0.09)$ & $* *$ & $100 \%$ \\
\hline Direct effect & 1.08 & $(0.61)$ & * & 77 & 0.20 & $(0.09)$ & ** & 79 \\
\hline Indirect, parenting strain & 0.06 & $(0.09)$ & & 5 & 0.01 & $(0.02)$ & & 6 \\
\hline Indirect, economic strain & 0.09 & $(0.06)$ & * & 7 & 0.02 & $(0.01)$ & * & 6 \\
\hline Indirect, education spending & 0.13 & $(0.10)$ & + & 9 & 0.02 & $(0.02)$ & + & 7 \\
\hline Indirect, exercise minutes & 0.05 & $(0.08)$ & & 4 & 0.01 & $(0.01)$ & & 3 \\
\hline Indirect, fruit or vegetable days & 0.02 & $(0.08)$ & & 1 & 0.00 & $(0.02)$ & & 1 \\
\hline Indirect, sleep hours & 0.01 & $(0.04)$ & & 0 & 0.00 & $(0.00)$ & & 0 \\
\hline
\end{tabular}

Source: Authors' tabulation based on the PSID.

Note: Results of parametric g-formula decomposition showing average difference in BMI (model 1: continuous $\mathrm{BMI}$; model 2: age standardized BMI z-score) for children ages thirteen through seventeen with family wealth at $+1 \mathrm{SD}$ versus $1 \mathrm{SD} ; \mathrm{n}=2,271 ; 5,411$ person-years.

${ }^{+} p<.10 ;{ }^{*} p<.05 ;{ }^{* *} p<.01$

the total direct contribution of household wealth to the adolescent BMI disparity as well as the contributions of our time-varying mechanisms (including the stress, behavioral, and spending measures) to the BMI disparity. Model 1 of table 3 shows results of the continuous BMI models, and model 2 shows results of the age-specific BMI z-score models.

Consistent with results from table 2 and table A1, results in models 1 and 2 of table 3 show that household wealth is strongly associated with adolescent BMI, as indicated by the parameter estimate of the total effect (model 1: $-1.39, p<.01$; model 2: $-0.25, p<.01$ ). For both outcomes, the majority of the estimated total effect of wealth on the adolescent BMI disparity operates directly (77 percent for continuous BMI in model 1 and 79 percent for BMI z-score in model 2). Together, these estimates indicate that most of the association of household wealth exposures across childhood and adolescence on adolescent BMI is not accounted for by our stress, spending, and behavioral mechanisms. Results from table 3 indicate that roughly 20 percent of the effect of household wealth operates indirectly through the hypothesized mechanisms. In particular, familial economic strain is a key indirect pathway linking household wealth to adolescent BMI (7 and 6 percent of the total wealth effect in models 1 and 2, respectively). Household wealth also operates indirectly through education spending, with 9 and 7 percent of the total effect of wealth on the adolescent BMI and obesity risk explained by household spending on educationrelated expenses, respectively. Importantly, results from models 1 and 2 provide little evidence that child health behaviors (exercise, diet, or sleep) mediate the association between household wealth and child BMI.

\section{DISCUSSION}

A large and growing body of research links wealth and adult health (Boen, Keister, and Aronson 2020; Boen and Yang 2016). Still, despite slow rates of wealth accumulation and growing wealth inequality among households with children (Gibson-Davis and Percheski 2018), little is known about whether or how household wealth shapes child health. In particular, we know little from research about how household wealth and child BMI are related. Given that BMI is a critical indicator of child health and well-being that has implications for health and other outcomes across the life course, this gap is particularly salient. Merging rich, longitudinal data from the CDS, TAS, and the main file of the PSID, this study is among the first to examine the association between household wealth and childhood BMI and to assess the direct and indirect pathways linking wealth to child BMI and obesity risk.

This study makes three key contributions to the literatures on wealth and child BMI. First, 
our study shows that household wealth is an important-yet largely understudied-correlate of child BMI, net of other indicators of family social and economic context, including maternal education, household income, child health insurance status, and parental marital status. Results shown in model 2 of table 2 and table A1 demonstrate that household wealth is negatively associated with child BMI and obesity risk, such that increases in household wealth are associated with lower childhood BMIs and BMI z-scores. In table 3 , results similarly show a strong total cumulative effect of household wealth on BMI and obesity risk in adolescence. Studies across disciplines provide evidence of a socioeconomic gradient in child health (Larson and Halfon 2010; Martinson and Reichman 2016), but little research to date has assessed the role of wealth in child health inequality. Findings from this study suggest that, net of widely used indicators of household SES such as income and parental education, wealth provides health protections to children in ways that shape trajectories of health across childhood and adolescence. Given that childhood BMI has been positively associated with premature morbidity and mortality in adulthood (Park et al. 2012; Reilly et al. 2003), our findings on the robust links between wealth and child BMI indicate that childhood wealth exposures may play a critical role in shaping life course patterns of population health inequality.

Second, our results show that total household wealth is an essential correlate of child BMI that is largely consistent across the components of net worth. That is, the association between the various assets and debts that make up total net worth are generally associated with childhood BMI in consistent ways. One potential exception is that home equity is negatively associated with child BMI. Home equity may affect child health directly by shaping familial financial security, but it may also relate to health indirectly by serving as a proxy for unmeasured indicators of social status such as homeownership or school or neighborhood quality. Still, results from models 4 and 5 of table 2 and table A1 suggest that homeownership and home value-which are perhaps better proxies of neighborhood context than home equity-were not associated with child BMI. Still, results also showed that none of the other wealth measures-including debt, stocks, checking and savings, and other assets-is associated with child BMI. Although research on adults shows that the components of wealth relate to health in different ways (Boen, Keister, and Aronson 2020), they do not for childhood BMI. It is possible that though children are largely unaware of any particular dimension of their household finances, the overall status of their family's financial stability and wellbeing-as captured by measures of total net worth-holds great bearing on their day-to-day lives in ways that matter for health. Altogether, findings from this study point to the important but largely understudied role of household wealth in shaping the emergence and divergence of child health disparities.

Third, findings from this study offer new evidence of the mechanisms linking household wealth to childhood BMI. Assessing and parameterizing the links between household wealth and child health presents tremendous empirical challenges in part because of the difficulties related to properly accounting for the complex interplay between time-varying confounding and mediation. As shown in figure 1, we use the recently developed $\mathrm{g}$-formula simulation to model the pathways through which household wealth affects child BMI directly as well as how its impacts might accumulate and manifest visà-vis other time-varying characteristics across childhood. Results from table 3 show that household wealth contributes to child BMI and obesity risk both directly and indirectly in several ways. In particular, results from the parametric g-formula analyses show that household wealth relates to child BMI over time by shaping parental economic strain and patterning household spending on education. We hypothesized that family stress would be an important indirect mechanism linking household wealth to child health. Research indicates that parents and caregivers under high levels of economic stress need to expend tremendous time and energy to managing the many social, emotional, and financial challenges associated with having few financial resources. In addition to shaping parental behavior, high levels of familial economic strain may also affect child BMI directly by chronically up-regulating physiological 
stress response systems in ways that promote weight gain and the redistribution of bodily fat stores (Scott, Melhorn, and Sakai 2012). Our results show that parental economic stress accounted for 7 percent and 6 percent of the association between household wealth and BMI and BMI z-score, respectively.

We also find that household wealth shaped BMI in childhood and adolescence by affecting household spending on education. Preliminary analyses also included other household consumption mechanisms, including spending on housing, childcare, and health spending, but none was significantly related to child BMI. Households with more wealth have more capital to invest in their children's education in ways that could shape child BMI through a variety of human capital (for example, increased knowledge, self-efficacy) and environmental exposure (healthier food options, opportunities for physical activity) pathways that we are unable to examine here but offer opportunities for future research.

Perhaps surprisingly, results in table 3 show that none of the hypothesized child behavioral mechanisms links household wealth to child health. Although health behaviors such as diet and physical activity garner much attention in policy and intervention discussions related to the childhood obesity epidemic, our results show that these behavioral factors do not help explain the wealth gradient in child BMI. Instead, results show that most of the association between household wealth and child BMI was unaccounted for by our mechanisms, including the indicators of stress, spending, and child health behaviors. These findings suggest that future research should continue to theorize and empirically test how household wealth shapes family life in ways that matter for child health and well-being, including how other meso-level environmental factors and indicators-such as school quality and neighborhood context-might undergird the links between wealth and child health. Future research should also consider the factors that can buffer against the health-harming effects of low wealth among children.

Taken together, this study provides new knowledge of the role of wealth inequality in producing population health disparities beginning early in the life course. Our findings add to the large and growing body of research showing strong, prospective associations between wealth and health that extend to the early life period. The results demonstrate that the wealth gradient in health emerges early in the life course, long before individuals begin to accumulate their own assets and debts. Levels of wealth inequality are particularly stark among child households in the United States (GibsonDavis and Hill 2021, this issue; Gibson-Davis and Percheski 2018), and the findings presented here show that this inequality greatly affects the development and divergence of health disparities among America's children. Future research should build on the findings presented here to consider how long-term trajectories of household wealth shape child health. Given stark racial differences in household wealth, our findings also suggest that wealth inequality likely also plays an essential role in the early-life emergence and intergenerational and historical persistence of racial health inequities. Findings from this project support the call for policy and intervention efforts to focus on building wealth among households with children as a way to promote child health equity. Efforts aimed at building and redistributing wealth are not solely economic in nature; wealth policies are health policies with important consequences for the health and well-being of children. 


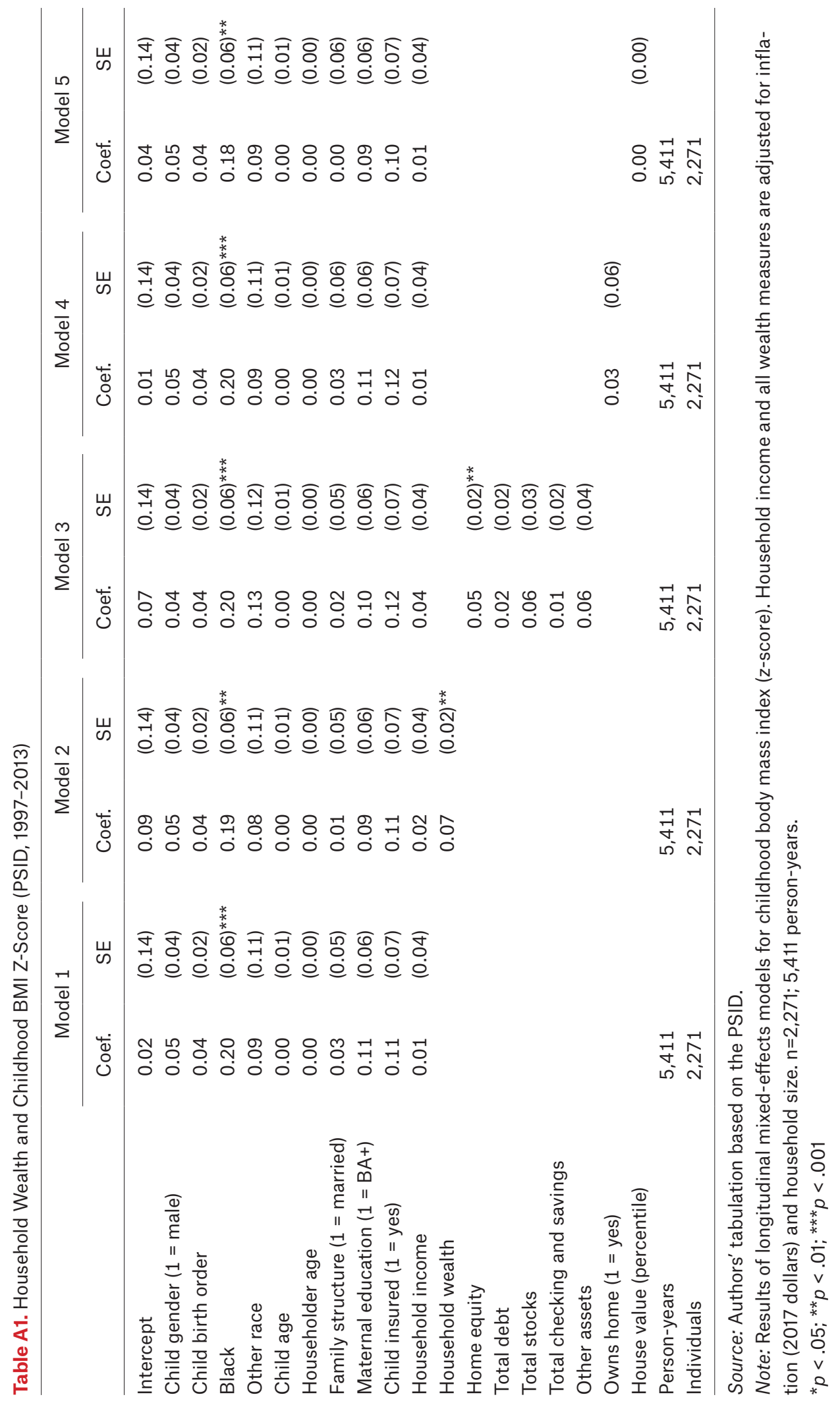




\section{REFERENCES}

Assari, Shervin. 2018. "Health Disparities Due to Diminished Return Among Black Americans: Public Policy Solutions." Social Issues and Policy Review 12(1): 112-45.

Berger, Lawrence M., and Jason N. Houle. 2019. "Rising Household Debt and Children's Socioemotional Well-Being Trajectories." Demography 56(4): 1273-301.

Boen, Courtney. 2016. “The Role of Socioeconomic Factors in Black-White Health Inequities Across the Life Course: Point-in-Time Measures, LongTerm Exposures, and Differential Health Returns." Social Science \& Medicine 170 (December): 63-76.

- 2020. “Death by a Thousand Cuts: Stress Exposure and Black-White Disparities in Physiological Functioning in Late Life." Journals of Gerontology: Series B 75(9): 1937-950.

Boen, Courtney, Lisa A. Keister, and Brian Aronson. 2020. "Beyond Net Worth: Racial Differences in Wealth Portfolios and Black-White Health Inequality Across the Life Course." Journal of Health and Social Behavior 61(2): 153-69.

Boen, Courtney, and Y. Claire Yang. 2016. “The Physiological Impacts of Wealth Shocks in Late Life: Evidence from the Great Recession." Social Science \& Medicine 150 (February): 221-30.

Bond Huie, Stephanie A., Patrick M. Krueger, Richard G. Rogers, and Robert A. Hummer. 2003. "Wealth, Race, and Mortality." Social Science Quarterly 84(3): 667-84.

Boshara, Ray, William R. Emmons, and Bryan J. Noeth. 2015. "The Demographics of Wealth-How Age, Education and Race Separate Thrivers from Strugglers in Today's Economy." In Demographics of Wealth. St. Louis, Mo.: Federal Reserve Bank of St. Louis.

Bronfenbrenner, Urie, and Stephen J. Ceci. 1994. "Nature-Nurture Reconceptualized in Developmental Perspective: A Bioecological Model." Psychological Review 101(4): 568-86.

Case, Anne, Darren Lubotsky, and Christina Paxson. 2002. "Economic Status and Health in Childhood: The Origins of the Gradient." American Economic Review 92(5): 1308-34.

Cesarini, David, Erik Lindqvist, Robert Östling, and Björn Wallace. 2016. “Wealth, Health, and Child Development: Evidence from Administrative Data on Swedish Lottery Players." Quarterly Journal of Economics 131(2): 687-738.
Chen, Edith, Andrew D. Martin, and Karen A. Matthews. 2006. "Socioeconomic Status and Health: Do Gradients Differ Within Childhood and Adolescence?" Social Science \& Medicine 62(9): 2161-70.

Currie, Janet, and Mark Stabile. 2003. "Socioeconomic Status and Child Health: Why Is the Relationship Stronger for Older Children?" American Economic Review 93(5): 1813-23.

Darity, William A., Jr., and A. Kirsten Mullen. 2020. From Here to Equality: Reparations for Black Americans in the Twenty-First Century. Chapel Hill: University of North Carolina Press.

Deaton, Angus S., and Christina H. Paxson. 1998. "Aging and Inequality in Income and Health." American Economic Review 88(2): 248-53.

Erixson, Oscar. 2017. "Health Responses to a Wealth Shock: Evidence from a Swedish Tax Reform." Journal of Population Economics 30(4): 1281-336.

Garasky, Steven, Susan D. Stewart, Craig Gundersen, Brenda J. Lohman, and Joey C. Eisenmann. 2009. "Family Stressors and Child Obesity." Social Science Research 38(4): 755-66.

Gaydosh, Lauren, Kristen M. Schorpp, Edith Chen, Gregory E. Miller, and Kathleen Mullan Harris. 2018. “College Completion Predicts Lower Depression But Higher Metabolic Syndrome Among Disadvantaged Minorities in Young Adulthood." Proceedings of the National Academy of Sciences 115(1): 109-14.

Gibson-Davis, Christina, and Heather D. Hill. 2021. "Childhood Wealth Inequality in the United States: Implications for Social Stratification and Well-Being." RSF: The Russell Sage Foundation Journal of the Social Sciences 7(3): 1-26. DOI: 10.7758/RSF.2021.7.3.01.

Gibson-Davis, Christina, Lisa A. Keister, and Lisa Genettian. 2020. "Net Worth Poverty in Child Households by Race and Ethnicity, 1989-2019." Journal of Marriage and Family. Published online November 26. DOI: 10.1111/jomf.12742.

Gibson-Davis, Christina M., and Christine Percheski. 2018. "Children and the Elderly: Wealth Inequality Among America's Dependents." Demography 55(3): 1009-32.

Hamilton, Darrick, and William Darity Jr. 2010. “Can 'Baby Bonds' Eliminate the Racial Wealth Gap in Putative Post-Racial America?" Review of Black Political Economy 37(3-4): 207-16.

Isong, Inyang A., Sowmya R. Rao, Marie-Abèle Bind, 
Mauricio Avendaño, Ichiro Kawachi, and Tracy K. Richmond. 2018. "Racial and Ethnic Disparities in Early Childhood Obesity." Pediatrics 141(1): e20170865. DOI: 10.1542/peds.2017-0865.

Keil, Alexander P., Jessie K. Edwards, David R. Richardson, Ashley I. Naimi, and Stephen R. Cole. 2014. “The Parametric G-Formula for Time-toEvent Data: Towards Intuition with a Worked Example." Epidemiology 25(6): 889-97.

Keister, Lisa A. 2014. “The One Percent." Annual Review of Sociology 40(1): 347-67.

Killewald, Alexandra, Fabian T. Pfeffer, and Jared N. Schachner. 2017. "Wealth Inequality and Accumulation." Annual Review of Sociology 43(1): 379-404.

Kochnar, Rakesh, and Anthony Cilluffo. 2017. "How Wealth Inequality Has Changed in the U.S. Since the Great Recession, by Race, Ethnicity and Income." Fact Tank (Pew Research Center blog), November 1. Accessed December 16, 2020. http://www.pewresearch.org/fact-tank/2017/11 /01/how-wealth-inequality-has-changed-in-the -u-s-since-the-great-recession-by-race-ethnicity -and-income.

Kuh, Diana, Yoav Ben-Shlomo, John Lynch, Johan Hallqvist, and Chris Power. 2003. "Life Course Epidemiology." Journal of Epidemiology and Community Health 57(10): 778-83.

Larson, Kandyce, and Neal Halfon. 2010. “Family Income Gradients in the Health and Health Care Access of US Children." Maternal and Child Health Journal 14(3): 332-42.

Marmot, Michael. 2004. "Status Syndrome." Significance 1(4): 150-54.

Martinson, Melissa L., and Nancy E. Reichman. 2016. "Socioeconomic Inequalities in Low Birth Weight in the United States, the United Kingdom, Canada, and Australia." American Journal of Public Health 106(4): 748-54.

McCurdy, Karen, Kathleen S. Gorman, and Elizabeth Metallinos-Katsaras. 2010. “From Poverty to Food Insecurity and Child Overweight: A Family Stress Approach." Child Development Perspectives 4(2): 144-51.

Naimi, Ashley I., Stephen R. Cole, and Edward H. Kennedy. 2017. "An Introduction to G Methods." International Journal of Epidemiology 46(2): 75662.

Oliver, Melvin, and Thomas Shapiro. 2013. Black Wealth/White Wealth: A New Perspective on Racial Inequality, 2nd ed. New York: Routledge.
Park, Min Hae, Catherine Falconer, Russell Viner, and Sanjay Kinra. 2012. "The Impact of Childhood Obesity on Morbidity and Mortality in Adulthood: A Systematic Review." Obesity Reviews 13(11): 985-1000.

Pearlin, Leonard I. 1989. "The Sociological Study of Stress." Journal of Health and Social Behavior 30(3): 241-56.

Pearlin, Leonard I., Joseph T. Mullan, Shirley J. Semple, and Marilyn M. Skaff. 1990. “Caregiving and the Stress Process: an Overview of Concepts and Their Measures." The Gerontologist 30(5): 58394.

Pfeffer, Fabian T., Sheldon Danziger, and Robert F. Schoeni. 2013. "Wealth Disparities Before and After the Great Recession." Annals of the American Academy of Political and Social Science 650(1): 98-123.

Piketty, Thomas. 2013. Capital in the Twenty-First Century. Cambridge, Mass.: Harvard University Press.

Pollack, Craig E., Sekai Chideya, Catherine Cubbin, Brie Williams, Mercedes Dekker, and Paula Braveman. 2007. "Should Health Studies Measure Wealth?: A Systematic Review." American Journal of Preventive Medicine 33(3): 250-64.

Powell, Lisa M., and Yanjun Bao. 2009. "Food Prices, Access to Food Outlets and Child Weight." Economics \& Human Biology 7(1): 64-72.

Raudenbush, Stephen W., and Anthony S. Bryk. 2002. Hierarchical Linear Models: Applications and Data Analysis Methods. Thousand Oaks, Calif.: Sage Publications.

Reilly, John J., E. Methven, Zoe C. McDowell, Belinda Hacking, D. Alexander, Laura Stewart, and Christopher J. H. Kelnar. 2003. "Health Consequences of Obesity." Archives of Disease in Childhood 88(9): 748-52.

Schmeer, Kammi K. 2012. "Family Structure and Obesity in Early Childhood." Social Science Research 41(4): 820-32.

Scott, Karen A., Susan J. Melhorn, and Randall R. Sakai. 2012. "Effects of Chronic Social Stress on Obesity." Current Obesity Reports 1(1): 16-25.

Shaw, Benjamin A., and Neal Krause. 2002. "The Impact of Salient Role Stress on Trajectories of Health in Late Life Among Survivors of a SevenYear Panel Study: Analyses of Individual Growth Curves." International Journal of Aging and Human Development 55(2): 97-116.

Spilerman, Seymour. 2000. "Wealth and Stratifica- 
tion Processes." Annual Review of Sociology 26(1): 497-524.

Tamayo, Teresa, Christian Herder, and Wolfgang Rathmann. 2010. "Impact of Early Psychosocial Factors (Childhood Socioeconomic Factors and Adversities) on Future Risk of Type 2 Diabetes, Metabolic Disturbances and Obesity: A Systematic Review." BMC Public Health 10(1): 525.

Tate, Eleanor B., Wendy Wood, Yue Liao, and Genevieve F. Dunton. 2015. “Do Stressed Mothers Have Heavier Children? A Meta-Analysis on the Relationship Between Maternal Stress and Child Body Mass Index." Obesity Reviews 16(5): 351-61. van Buuren, Stef, and Karin Groothuis-Oudshoorn.
2011. “MICE: Multivariate Imputation by Chained Equations in R." Journal of Statistical Software 45(1): 1-67.

Ward, Zachary J., Michael W. Long, Stephen C. Resch, Catherine M. Giles, Angie L. Cradock, and Steven L. Gortmaker. 2017. “Simulation of Growth Trajectories of Childhood Obesity into Adulthood." New England Journal of Medicine 377(22): 2145-53.

Yang, Y. Claire, Karen Gerken, Kristen Schorpp, Courtney Boen, and Kathleen Mullan Harris. 2017. "Early-Life Socioeconomic Status and Adult Physiological Functioning: A Life Course Examination of Biosocial Mechanisms." Biodemography and Social Biology 63(2): 87-103. 